\title{
LA BONNE ADMINISTRATION EN DROIT COMMUNAUTAIRE ET LE CODE EUROPÉEN DE BONNE CONDUITE ADMINISTRATIVE
} Joana Mendes

\author{
Ecole nationale d'administration (ENA) | « Revue française d'administration \\ publique »
}

2009/3 n 131 | pages 555 à 571

ISSN 0152-7401

ISBN 9782909460147

Article disponible en ligne à l'adresse :

http://www.cairn.info/revue-francaise-d-administrationpublique-2009-3-page-555.htm

\section{Pour citer cet article :}

Joana Mendes, « La bonne administration en droit communautaire et le code européen de bonne conduite administrative », Revue française d'administration publique 2009/3 ( $\mathrm{n}^{\circ} 131$ ), p. 555-571.

DOI 10.3917/rfap.131.0555

Distribution électronique Cairn.info pour Ecole nationale d'administration (ENA).

(C) Ecole nationale d'administration (ENA). Tous droits réservés pour tous pays.

La reproduction ou représentation de cet article, notamment par photocopie, n'est autorisée que dans les limites des conditions générales d'utilisation du site ou, le cas échéant, des conditions générales de la licence souscrite par votre établissement. Toute autre reproduction ou représentation, en tout ou partie, sous quelque forme et de quelque manière que ce soit, est interdite sauf accord préalable et écrit de l'éditeur, en dehors des cas prévus par la législation en vigueur en France. Il est précisé que son stockage dans une base de données est également interdit. 


\title{
LA BONNE ADMINISTRATION EN DROIT COMMUNAUTAIRE ET LE CODE EUROPÉEN DE BONNE CONDUITE ADMINISTRATIVE ${ }^{1}$
}

\author{
Joana MENDES
}

Chercheur post-doctoral, département de droit, Université d'Amsterdam

\begin{abstract}
Résumé
Le code de bonne conduite administrative constitue une source importante pour comprendre le sens du principe et concept de bonne administration en droit administratif européen. Il englobe en effet certains aspects qui ont tendance à être négligés dans la jurisprudence des juridictions européennes ainsi que par les chercheurs en droit européen. De plus, contrairement à ce que les récents développements ont laissé présager - c'est-à-dire le fait que la Commission refuse de présenter une proposition de règlement européen qui donnerait une force obligatoire aux dispositions du code - celui-ci demeure pertinent pour envisager les évolutions juridiques possibles de la bonne administration. Le présent article met en avant les raisons et le sens du lien entre le code et l'article 41 de la Charte des droits fondamentaux, analyse la complexité et les incertitudes entourant le concept de «bonne administration», souligne les interconnexions entre ses aspects juridiques et extra-juridiques. Il aborde également les rapports du code avec des développements antérieurs du droit communautaire. Il met en lumière sa valeur ajoutée juridique et les fonctions qu'il exerce à l'heure actuelle tout en considérant les évolutions juridiques qui pourraient en découler.
\end{abstract}

\begin{abstract}
- Good administration in community law and the European Code of Good Administrative Behaviour - The Code of Good Administrative Behaviour is an important source for understanding the principle and concept of good administration in European administrative law, since it encompasses certain aspects that tend to be overlooked by the case law of the European Courts and European law scholars. Furthermore, contrary to what recent developments would lead people to expect - namely, the fact that the Commission is refusing to put forward a proposal for a European regulation that would make the Code's provisions binding - it is still a relevant tool for mapping potential legal developments in good administration. This article explains the reasons and meaning of the link between the Code and Article 41 of the Charter of Fundamental Rights, analyses the complex issues and areas of uncertainty surrounding the good administration concept, and highlights the interconnections between its different legal and non-legal facets. In addition, it demonstrates how these different aspects are reflected in the Code and shows how it relates to previous developments in community law. It highlights its added legal value and the functions it currently performs, while also considering further developments that may occur as a result.
\end{abstract}

1. Traduit par Dov Jacobs. 
Le code européen de bonne conduite administrative (ci-après, le code) fut proposé en 1999 par le Médiateur européen aux institutions, agences et organes européens. Le but était qu'il soit un modèle pour l'adoption de codes de bonne conduite de ces derniers, en vue d'améliorer les standards de bonne administration et les rapports entre l'administration européenne et le public ${ }^{2}$. Sa reconnaissance par le Parlement européen en 2001 a renforcé sa légitimité politique. Par ailleurs, son association à l'article 41 de la Charte des droits fondamentaux de l'Union européenne a ouvert la voie à son éventuelle reconnaissance constitutionnelle en raison de sa vocation à expliciter le droit à une bonne administration y étant contenu ${ }^{3}$. Plus tard, dans l'introduction à la version grand public du code, le Médiateur a souligné le lien de la façon suivante : «Le code a pour vocation d'expliquer plus précisément ce que le droit à une bonne administration de la Charte devrait vouloir dire en pratique ${ }^{4}$.

Au préalable, il convient de garder à l'esprit que la définition de la bonne administration reste incertaine, que l'article 41 de la Charte a un caractère ouvert en ce sens que la liste des règles procédurales qu'il renferme n'est pas exhaustive, et que, si la Charte devient juridiquement obligatoire, des évolutions juridiques ne manqueront pas de se produire.

Ceci étant précisé, l'article 41 de la Charte a trois objectifs. Premièrement, il donne le sens de l'association du code à l'article 41. Deuxièmement, il tente de systématiser les différentes branches du concept de bonne administration présentes dans la jurisprudence ainsi que dans les décisions du Médiateur. Les différentes dimensions du concept de bonne administration seront analysées, y compris son acception en tant que droit subjectif. Nous soutiendrons que le code contribue à clarifier le contenu de la bonne administration en droit communautaire, mais il est erroné de considérer qu'il précise le contenu du droit subjectif à une bonne administration. L'analyse du contenu du code le confirmera. Troisièmement, l'article présente les fonctions actuelles du code et souligne sa possible contribution au développement du droit administratif européen.

\section{LE CODE, LA CHARTE ET LE «DROIT ADMINISTRATIF EUROPÉEN » : HISTOIRES MÊLÉES}

À l'origine, le code avait trois objectifs principaux. Il visait à formaliser les règles et principes en vertu desquels le Médiateur était en mesure d'évaluer les cas de mauvaise administration, à fournir un guide pour les institutions de la Communauté et les agences concernant leurs rapports avec le public, et à informer les citoyens « de leurs droits ainsi

2. Draft recommendation to the European institutions, bodies and agencies in the own initiative inquiry, OI/1/98/OV, 13 septembre 1999, paragraphes 1.1 à 1.3 .

3. Résolution A5-0245/2001, Résolution du Parlement européen sur le rapport spécial du Médiateur européen au Parlement européen faisant suite à une enquête de propre initiative sur l'existence, au sein de chaque institution ou organe communautaire, d'un code, accessible au public, relatif à la bonne conduite administrative (C5-0438/2000 - 2000/2212(COS)), 6 septembre 2001 ; voir modification 2.

4. L'article 41 de la Charte énumère de manière non exhaustive les droits et obligations suivants comme faisant partie du droit à une bonne administration: le droit de voir ses affaires traitées impartialement, équitablement et dans un délai raisonnable (paragraphe 1), le droit d'être entendu, d'accéder à son dossier et le devoir de l'administration de motiver ses décisions (paragraphe 2), ainsi que la responsabilité noncontractuelle de l'Union européenne (paragraphe 3) et des droits linguistiques. 
que des normes de gestion administrative au respect desquelles ils pourraient s'attendre ${ }^{5}$. Assez tôt, le code fut aussi associé au droit à une bonne administration contenu à l'article 41 de la Charte des droits fondamentaux. Le Médiateur lui-même, dans son discours à la Convention chargée de la rédaction de la Charte (ci-après la Convention), suggéra l'inclusion du droit à une bonne administration dans le document où il « devrait être érigé en principe ${ }^{6}$.

La décision d'adopter la Charte avait été prise au Conseil européen de Cologne en juin 1999. Le Médiateur y vit une opportunité d'insister sur l'importance des rapports entre l'administration européenne et le public ${ }^{7}$. De façon plus prosaïque, l'association du code à ce droit pouvait être un argument supplémentaire pour convaincre les institutions et les agences de se conformer aux recommandations du Médiateur sur l'adoption de codes de bonne conduite administrative. En fait, au moment du discours devant la Convention, les efforts du Médiateur en vue de l'adoption de codes de bonne conduite administrative par les institutions et les agences européennes n'avaient pas encore obtenu de résultats tangibles. En mars 2000, la Commission avait adopté un projet de code qui, selon le Médiateur, n'était pas conforme à ses recommandations. Par ailleurs, le Conseil et le Parlement européens avaient été réceptifs à l'initiative du Médiateur, sans pour autant donner suite à ses recommandations. Enfin, seulement deux agences avaient suivi les propositions du Médiateur sur ce point ${ }^{8}$.

Étant donné ces circonstances, en plus de proposer l'inclusion du droit à une bonne administration dans la Charte, le Médiateur décida de changer de stratégie : il soutint que les règles de bonne conduite européennes devraient être adoptées sous la forme d'un règlement ${ }^{9}$. Dans son discours devant la Convention, le Médiateur souligna l'importance de cette recommandation, insistant sur le fait que «pour la mise en œuvre pratique du principe [de bonne administration], il serait nécessaire d'adopter un règlement sur la bonne conduite administrative et un autre sur l'accès à l'information et aux documents $»{ }^{10}$. Bien que le Médiateur ait dès le départ défendu l'idée selon laquelle les institutions et organes

5. Rapport annuel du Médiateur européen (RA ME), 1998, p. 19. Avant-propos par le Médiateur européen au code européen de bonne conduite administrative, p. 4 (version en ligne publiée en septembre 2005). Tous les rapports annuels, décisions, projets de recommandations, discours et autres documents du Médiateur européen cités dans cet article sont disponibles à http://www.ombudsman.europa.eu. Le code contient des droits et obligations de nature procédurale (par exemple, le droit à un traitement juste et impartial, le droit d'être entendu, l'accès au dossier, l'obligation de motiver les décisions), des droits substantiels (par exemple, la protection des données personnelles), des principes généraux de droit administratif européen (par exemple, la proportionnalité) et des règles de conduite éthique et de bonnes prestations administratives (par exemple, la courtoisie).

6. Discours du Médiateur européen - audition publique sur le projet d'une Charte des droits fondamentaux de l'Union européenne, Bruxelles, 2 février $2000 ; R A M E, 2001$, p. 19.

7. Le contexte était favorable à une telle initiative. À la suite de la Commission Santer, le Premier Rapport du Comité d'Experts Indépendants sur les allégations de fraude, de mauvaise gestion et de népotisme à la Commission européenne (15 mars 1999) souligna que les normes minimales de standards de bonne conduite dans l'exercice de la fonction publique incluaient des règles de publicité, disponible à http:// www.europarl.europa.eu/experts/pdf/reportfr.pdf

8. L'Agence européenne pour l'évaluation des médicaments et le Centre de traduction des organes de l'Union européenne. Voir le Rapport spécial du Médiateur européen au Parlement européen, faisant suite à une enquête de propre initiative sur l'existence, au sein de chaque institution ou organe communautaire, d'un code, accessible au public, relatif à la bonne conduite administrative des fonctionnaires (OI/1/98/OV), d'avril 2000, partie B (Réponses aux projets de recommandations du Médiateur) et partie C (Analyse des réponses). Concernant les codes finalement adoptés par les institutions, voir note 41 infra.

9. Idem, partie $\mathrm{D}$, conclusions et recommandations $\mathrm{n}^{\text {os }} 4$ à 9. L'opinion du Parlement européen selon laquelle de telles règles devraient s'appliquer de la même manière à toutes les institutions et organes a pesé dans le choix du Médiateur (conclusion $\mathrm{n}^{\circ} 1$ ).

10. Discours, cit., note 6. 
adopteraient des actes contraignants contenant leurs règles de bonne conduite administrative, il s'agissait dans son esprit d'une décision adoptée par chaque institution ${ }^{11}$. En d'autres termes, ces actes juridiques seraient des actes des institutions régissant leur propre fonctionnement (l'équivalent de règles de procédure, avec une portée externe limitée) et non une loi clarifiant le contenu d'un droit fondamental. En ce sens, le changement de stratégie impliquait un changement dans la pertinence politique du code. Celui-ci est devenu un modèle pour la codification formelle des règles administratives européennes relatives à la concrétisation d'un droit fondamental.

Le Parlement européen prit en compte la recommandation du Médiateur. Dans la résolution où il approuva le code proposé par le Médiateur, il associa explicitement le code au droit à une bonne administration comme un droit du citoyen (reconnu à « chaque personne »), tout en appelant la Commission à soumettre une proposition de règlement, fondée sur l'article 308 du Traité CE, contenant un code de bonne conduite administrative ${ }^{12}$. Une attente forte était née de l'inclusion dans le Traité constitutionnel d'un article III-389 reproduit à l'article 298 du Traité sur le fonctionnement de l'Union européenne (ci-après TFUE) ${ }^{13}$. Cependant, elle fut récemment très atténuée par une déclaration du représentant de la Commission lors de la présentation du Rapport annuel du médiateur au Parlement européen, par laquelle la Commission indiqua qu'elle n'avait pas l'intention de faire une proposition en vue de transformer le code en règlement ${ }^{14}$.

La portée politique du code, ainsi que son influence sur les institutions ont été alors atteintes ${ }^{15}$. Il n'en reste pas moins que le code demeure une source importante pour appréhender les contours de la bonne administration en droit communautaire et pour percevoir ses possibles développements. Au préalable, nous défendrons l'idée que le code ne devrait pas être lu comme explicitant le contenu de l'article 41 de la Charte.

\section{La bonne administration : droit, principe ou standard de comportement ?}

Avant d'être consacré comme droit fondamental, la bonne administration avait été reconnue comme un principe général du droit par les juridictions européennes ${ }^{16}$. La doctrine a souligné le sens incertain et ambigu de ce principe. Plus particulièrement, elle a mis en lumière son absence d'existence autonome comme règle dans la jurisprudence. La

11. Draft recommendation, cit., note 2 , recommendation $\mathrm{n}^{\mathrm{o}} 3$.

12. Voir, modifications 1,2 , 4 et point 1 de la Résolution A5-0245/2001, cit., note 3. Sur les origines de la seconde suggestion, voir Médiateur européen, $R A M E, 2001$, p. 19.

13. RA ME, 2003, p. 30. L'article 298 est ainsi rédigé : «1. Dans l'accomplissement de leurs missions, les institutions, organes et organismes de l'Union s'appuient sur une administration européenne ouverte, efficace et indépendante. 2. Dans le respect du statut et du régime adoptés sur la base de l'article 336, le Parlement européen et le Conseil, statuant par voie de règlements conformément à la procédure législative ordinaire, fixent les dispositions à cet effet ».

14. Intervention de M. Piebalgs lors de la session plénière du 23 octobre 2008, http:// www.europarl.europa.eu/sides/getDoc.do ?type=CRE\&reference=20081023\&secondRef=ITEM-

004\&language $=E N$. L'auteur remercie Peter Bonnor qui a porté cet élément à son attention.

15. Ces dernières ont déjà tendance à suivre leurs propres standards de bonne conduite administrative ne correspondant pas toujours à ceux du code. Voir infra note 41 et page 19.

16. Voir les explications relatives à la Charte des droits fondamentaux (JOUE C 303/17, 14 décembre 2007). Les expressions «juridictions européennes » et «tribunaux européens » dans cet article renvoient à la Cour de justice des communautés européennes et au Tribunal de première instance des Communautés européennes. 
bonne administration est le plus souvent associée à d'autres principes, droits et devoirs ${ }^{17}$. On pourrait affirmer, à la lecture de la jurisprudence, qu'au cœur du principe se trouve l'obligation d'examiner avec précaution et impartialité les circonstances factuelles et juridiques spécifiques à chaque espèce ${ }^{18}$.

\section{Un droit au sens encore incertain}

La nouveauté de l'article 41 de la Charte réside dans le fait qu'il élève la bonne administration « en catégorie générale sous laquelle peuvent être subsumés un ensemble de droits subjectifs destinés à limiter l'arbitraire administratif dans l'Union » ${ }^{19}$. Il n'en reste pas moins que le sens du droit à une bonne administration demeure obscur. L'article 41 réunit sous un chapeau général quelques droits et obligations. Les raisons du choix de ceux-ci plutôt que d'autres semblent répondre à une logique plus pragmatique que normative. Suivant l'esprit de la Charte, ces droits et obligations correspondent à des règles qui étaient soit établies dans la jurisprudence des tribunaux européens, soit définies dans le traité (comme l'obligation de motiver les décisions et la responsabilité extracontractuelle) ou encore faisaient partie des principes procéduraux fondateurs de la Communauté (règles linguistiques) ${ }^{20}$. En ce qui concerne leurs contenus respectifs, la valeur ajoutée du fait d'avoir regroupé chacun de ces droits sous l'intitulé de droit à une bonne administration est incertaine, sauf l'intention d'établir ceux-ci comme des droits publics subjectifs à caractère fondamental ${ }^{21}$. En d'autres termes, le sens de ce «droit-chapeau » demeure incertain.

D'une certaine manière, le code a davantage compliqué le cadre terminologique et conceptuel de la bonne administration. Tout en voulant expliciter le contenu du droit à une bonne administration, il présente une série de règles éclectiques qui incluent des principes qui ont chacun une existence indépendante de la bonne administration (comme la proportionnalité et la non-discrimination) et des règles auparavant inconnues, à l'exception des avocats moins attentifs aux interventions du Médiateur (tel que le devoir d'être consciencieux, l'obligation d'être correct et courtois). Le code recouvre les deux aspects

17. Nehl (Hans Peter), Principles of Administrative Procedure in EC law, Oxford, Hart Publishing, 1999, pp. 15-20, 27-37 et aussi Nehl (Hans Peter), « Good administration as a procedural right and/or general principle » in Hofmann (Herwig C.H.) and Türk (Alexander H.), Legal Challenges in EU Administrative Law. Towards an Integrated Administration, Edward Elgar, 2009, pp. 322-351, p. 336 ; Simon (Denys), «Le principe de 'bonne administration' ou la 'bonne gouvernance'concrète » in Le droit de l'Union européenne en principes. Liber Amicorum en l'honneur de Jean Raux, Rennes, Éditions Apogée, 2006, pp. 155-176, p. 165 ; Trimarchi Banfi (Francesca), «Il diritto ad una buona amministrazione », in Chiti (Mario) e Greco (Guido) eds., Trattato di diritto amministrativo, Tomo I, $2^{\mathrm{e}}$ ed., Milano, Giuffrè, 2007, pp. 49-86, pp. 49-50.

18. Azoulai (Loïc), «Le principe de bonne administration », in Auby (J.-B.), Dutheil de La Rochère (Jacqueline) eds., Droit administratif européen, Bruxelles, Bruylant, 2007, pp. 493-518, pp. 496-511. Hofmann (Herwig), «Good administration in the EU law - a fundamental right ? », Bulletin des Droits de l'Homme, 2007, no 13, pp. 44-52, p. 48 ; Nehl (Hans Peter), « Good administration as a procedural right... », op. cit., pp. 329, 333 and 336.

19. Azoulai (Loïc), op. cit., p. 504. Sur ce point, voir également Ka\&nacute ;ska (K.), « Towards administrative human rights in the EU. Impact of the Charter of Fundamental Rights », European Law Journal, 2004, Vol. 10, n 3, pp. 296-326, p. 300; Dutheil de la Rochère (Jacqueline), «The EU Charter of Fundamental Rights, not binding but influential : the example of good administration », in Arnull (A.), Eckhout (P.) et Tridimas (T.) eds., Continuity and change in EU law. Essays in honour of Sir Francis Jacobs, Oxford : Oxford University Press, 2007, pp. 157-171, p. 168 ; Banfi, op. cit., pp. 52-53.

20. C'est-à-dire l'article 2 du règlement $\mathrm{n}^{\circ} 1 / 58$ du Conseil portant fixation du régime linguistique de la Communauté économique européenne (JO L 17/385, 6.10.1958). Plus généralement sur ce point voir, Dutheil de la Rochère, op. cit. p. 167.

21. Sur l'intérêt de considérer la bonne administration comme un droit fondamental, voir Azoulai, loc. op. ult. cit. 
des pouvoirs de contrôle du Médiateur, traitant à la fois du contrôle de légalité et du contrôle des aspects extra-juridiques de l'action administrative ${ }^{22}$. En même temps, il met en lumière la spécificité de l'expression de bonne administration, comme nous allons à présent le montrer.

La bonne administration est un concept complexe et protéiforme. Il caractérise un modèle d'administration qui vise à satisfaire de façon adéquate et efficace l'intérêt public, en respectant les droits et les intérêts des personnes à qui il est adressé, et en étant au service de la communauté de manière à promouvoir la confiance envers l'action administrative et son acceptation. En ce sens, la bonne administration a un objectif programmatique important, identifiable lorsque les juridictions européennes considèrent que le respect de certaines règles, principes ou droits est «dans l'intérêt d'une bonne administration », « répond aux exigences d'une bonne administration », ou plus précisément, est «dans l'intérêt d'une bonne administration des règles fondamentales du Traité » ${ }^{23}$. La réalisation de ces objectifs de bonne administration requiert la combinaison de règles à la fois juridiques et extra-juridiques. Cela fut souligné par l'Avocat général Slynn dans ses conclusions maintes fois citées de l'affaire Tradax et a été confirmé par les tribunaux communautaires, lorsqu'ils considèrent, par exemple, qu'un comportement regrettable est passible d'enfreindre le principe de bonne administration mais n'affecte pas la légalité de la décision ( $A B B$ Asea Brown Boveri) ou que les règles visant à satisfaire une bonne administration ne constituent pas forcément des garanties procédurales directement invocables par les particuliers (Aseprofar and Edisa) ${ }^{24}$.

\section{Une interconnexion de différentes garanties juridiques et extra-juridiques}

Sur cette base, on peut décrire la bonne administration comme étant composée de plusieurs niveaux interconnectés. Premièrement, elle comprend des garanties procédurales qui visent principalement à protéger les droits substantiels des personnes dans leurs relations avec l'administration européenne. La violation de ces droits peut donner lieu à une action en justice et éventuellement conduire à l'annulation de l'acte vicié ou à l'octroi d'une compensation pour dommages subis. Il s'agit du dénominateur commun de l'article 41 de la Charte. Dans la mesure où cet article élève la bonne administration au rang d'un droit public subjectif, il délimite les aspects de la bonne administration qui peuvent principalement être perçus comme tel. Deuxièmement, la bonne administration recouvre les règles juridiques qui structurent l'exercice de la fonction administrative par référence aux intérêts objectifs d'une bonne application des règles du Traité et à la définition de l'intérêt public (par exemple l'obligation d'un examen prudent et impartial dans la mesure où elle a une portée plus large que la considération d'affaires privées). Ces règles opèrent aussi comme des garanties procédurales, mais leur objectif principal est d'encadrer l'exercice du pouvoir discrétionnaire à la lumière de la satisfaction de l'intérêt public dans chaque espèce

22. Craig (P.), EU Administrative Law, Oxford : OUP, 2006, pp. 852-3. Sur ce point, voir « Legality and good administration : is there a difference ? », Discours du Médiateur européen, P. Nikiforos Diamandouros, au Sixième Séminaire des Médiateurs nationaux des États membres de l'UE et des pays candidats sur Rethinking Good Administration in the European Union, Strasbourg, 15 octobre 2007.

23. CJCE, 6 mars 2003, Interporc c. Commission, aff. C-41/00 P, Rec. p. I-2125, pt 48 ; TPI, 8 mars 2005, Vlachaki c. Commission, aff. T-277/03, Rec. p. I-A-57, pt 64 ; TPI, 28 novembre 2008, Hôtel Cipriani c. Commission, aff. jointes T-254/00, T-270/00, T-277/00, pt 210.

24. Conclusions de l'Avocat général Slynn dans CJCE, 15 mars 1984, Tradax c. Commission, aff. 64/82, Rec. pp. 1385-6 ; TPI, 20 mars 2002, ABB Asea Brown Boveri Ltd c. Commission, aff. T-31/99, Rec. p. II-1881, pt 104 ; TPI, 19 septembre 2005, Aseprofar et Edifa c. Commission, aff. T-247/04, Rec. p. II-3449, pt 56. 
ainsi que de contrôler les actes de l'administration ${ }^{25}$. Les règles extra-juridiques constituent le troisième niveau de la bonne administration. Elles définissent des standards de comportement visant à assurer le bon fonctionnement des services administratifs fournis au public, assurant et démontrant leur efficacité et leur qualité. Naturellement, cet aspect de la bonne administration est essentiellement couvert par les interventions du Médiateur. En effet, il a constamment rappelé que « les principes de bonne administration [imposent] aux institutions et organes communautaires non seulement de respecter les obligations juridiques qui leur incombent, mais également de se montrer serviables et de veiller au traitement juste des citoyens et au plein exercice de leurs droits ${ }^{26}$. Les juridictions européennes approuvent également ce point de vue ${ }^{27}$. Récemment, la nature protéiforme de la bonne administration a été réaffirmée dans Dynamiki, où le Tribunal de première instance a estimé qu'une réponse rapide à des requêtes en l'absence d'une obligation légale «fait preuve d'un niveau de diligence propre à une bonne administration ». De plus, il affirma que le non-respect d'une obligation (légale) d'agir dans un délai raisonnable impliquait, dans les circonstances de l'espèce, que la Commission avait violé son obligation de diligence et de bonne administration. Cependant, cette violation n'avait pas « restreint la possibilité de la requérante de faire valoir ses droits devant le Tribunal » et ne devait donc pas conduire à l'annulation de la décision ${ }^{28}$.

Dans une certaine mesure, cette systématisation à trois niveaux reflète la distinction entre les fonctions subjectives et objectives des droits procéduraux - protection de droits subjectifs substantiels et poursuite de l'intérêt public. Alors qu'il est utile de noter que de nombreuses règles assurent les deux fonctions ${ }^{29}$, cette distinction pertinente en droit communautaire et a été renforcée par la Charte. En effet, le Tribunal de première instance a affirmé que le principe de bonne administration ne conférait pas de droits aux individus, sauf quand « il constitu[ait] l'expression de droits spécifiques » tels que ceux mentionnés à l'article $41^{30}$. Par ailleurs, le droit à une bonne administration a tendance à être identifié aux garanties procédurales contenues dans l'article 41, à la fois par le Tribunal de première instance et par les avocats généraux (en l'absence de décision de la Cour de justice en lien avec ce droit) ${ }^{31}$. Une position similaire est adoptée par le Médiateur ${ }^{32}$. Significativement,

25. CJCE, 21 novembre 1991, Technische Universität München c. Hauptzollamt München-Mitte, aff. C-269/90, Rec. p. I-5469, pts 13 et 14.

26. $R A M E, 2005$, p. 39 ; RA ME, 2006, p. 37 ; RA ME, 2007, p. 33. Voir Discours, cit., note 22, plus particulièrement les parties 3 et 4 .

27. Voir, plus particulièrement, ABB Asea Brown Boveri, cit., note 24.

28. TPI, 12 novembre 2008, Evropaïki Dynamiki c. Commission, aff. T-59/05, pts 150, 156 et 159.

29. Azoulai, op. cit., pp. 507-508.

30. TPI, 4 octobre 2006, Hans-Martin Tillack c. Commission, aff. T-193/04, Rec. p. II-3995, pt 127 ; TPI, 13 novembre 2008, Société de Plantations de Mbanga SA (SPM) c. Council and Commission, aff. T-128/05, pt 127.

31. Par ex. TPI, $1^{\text {er }}$ août 2003, Technische Glaswerke Ilmenau c. Commission, aff. T-378/02 R, Rec. p. I-2921, pt 65 ; conclusions de l'avocat général Kokott du 22 janvier 2009, Christopher Mellor c. Secretary of State for Communities and Local Government, aff. C-75/08, pt 24 (obligation de motiver) ; conclusions de l'avocat général Ruiz-Jarabo Colomer du 27 novembre 2007, SECAP c. Comune di Torino and Santorso c. Comune di Torino, aff. jointes C-147/06 et C-148/06, Rec. p. I-3565, pt 50 (droit d'être entendu) ; conclusions de l'avocat général Sharpston du 25 octobre 2007, Varec c. Belgium, aff. C-450/06, Rec. p. I-581, pt 43 (accès au dossier dans le contexte du secret des affaires) ; conclusions de l'avocat général Mengozzi du 16 novembre 2006, Commission c. Netherlands, aff. C-523/04, Rec. p. I-3267, pt 59, note 36 (délai raisonnable pour rendre une decision) ; conclusions de l'avocat général Poiares Maduro du 21 octobre 2004, Commission c. T-Mobile Austria GmbH, aff. C-141/02 P, Rec. p. I-1283, pt 56, contestant qu'un droit d'action individuel puisse découler de l'obligation de diligence et d'examen impartial.

32. Décision sur la plainte 1999/2007/FOR contre l'Office pour l'Harmonisation du Marché Intérieur, 26 juin 2008, pt 2.6 (droit d'être entendu) ; décision sur la plainte 3502/2004/GG contre la Commission 
dans les procédures dans lesquelles les plaignants ont invoqué le droit à une bonne administration en l'associant avec des règles autres que celles de l'article 41, le Médiateur a préféré faire référence dans sa décision au «principe » de bonne administration ${ }^{33}$. Dans une décision de cet ordre, le Médiateur, en lien avec la même plainte, a évoqué le « droit » à une bonne administration pour ce qui avait rapport aux arguments liés aux droits contenus dans l'article 41, mais a mentionné le « principe » de bonne administration lorsqu'il faisait référence à d'autres règles ${ }^{34}$. Cela semble indiquer que toutes les règles qui peuvent être incluses dans un principe de bonne administration ne seront pas forcément considérées comme faisant partie du droit à une bonne administration, la possibilité qu'elles puissent être vues comme des droits publics subjectifs, et donc être à la base de plaintes juridiques individuelles, étant éloignée ou indésirable. En conséquence, se concentrer sur la bonne d'administration sous l'angle des droits publics subjectifs a introduit une césure dans le concept de bonne administration entre une approche juridique plus stricte et une autre approche retenant un sens plus large, habituellement associé au principe de bonne administration, qui contient à la fois des règles juridiques et extra-juridiques. Bien que la perception des règles pouvant être conçues comme des droits procéduraux subjectifs puisse évoluer avec le temps, elles semblent pour le moment être limitées à l'énumération de l'article 41.

Soulignons que certaines règles de bonne administration appartiennent aux différents niveaux. L'accès à l'information, par exemple, peut être considéré comme un droit procédural - accès au dossier, prévu à l'article 41(2) alinéa premier ; droit d'accès aux documents en vertu de l'article 255 Traité CE (voir aussi l'article 15 (3) du Traité sur le fonctionnement de l'Union européenne) et règlement 1049/2001 - ou une règle extrajuridique - si l'information requise n'est pas couverte par ce règlement ou par les règles applicables à l'accès au dossier, mais dont la disponibilité est néanmoins considérée comme favorisant le modèle d'administration voulu (comme cela est indiqué à l'article 22 du code).

On peut soutenir que la caractéristique principale de la bonne administration réside dans la combinaison et le chevauchement partiel entre les questions de légalité et les aspects extra-juridiques de la bonne administration. Des règles spécifiques de bonne conduite administrative peuvent se dégager de cet enchevêtrement, qui permet de façon indirecte de renforcer les garanties accordées aux personnes en relation avec l'administration européenne dans les domaines qui ne ressortissent pas de la juridiction des tribunaux européens. Le Médiateur a mis cet aspect en lumière dans un discours récent où il souligna l'importance d'aller au-delà d'une simple évaluation de la légalité d'une action administrative ${ }^{35}$. Le code de bonne conduite administrative recouvre adéquatement les différentes ramifications de la bonne administration et indique certaines des règles qui peuvent découler d'une telle interaction. Il est donc en ce sens réducteur de considérer que le code ne fait qu'expliciter le droit à une bonne administration tel qu'envisagé dans l'article 41 de la Charte.

européenne, 8 avril 2008, pt 4.3 (obligation d'agir dans un délai raisonnable); décision sur la plainte 821/2003/JMA contre le Parlement européen, 22 septembre 2004, pt 1.4 (obligation de motivation) ; décision sur la plainte 1349/2003/JMA contre la Commission européenne, 7 juin 2004, pt 1.3 (obligation de motivation); décision sur la plainte 1100/2001/GG contre la Commission européenne, 5 mars 2002, pts 2.3, 2.4 et conclusion (obligation d'agir dans un délai raisonnable).

33. Décision sur la plainte 258/2007/(MNZ)RT contre la Commission européenne, 10 décembre 2007, pt 2.4 (manque de réponse en temps utile et absence d'excuses) ; décision sur la plainte 3398/2005/ELB contre la Commission européenne, 29 décembre 2006, pt 3.4 (cohérence).

34. Décision sur la plainte 1200/2003/OV contre le Conseil de l’Union européenne, 19 décembre 2003, pts 2.6 (droit d'être entendu), 4.3 (obligation de réponse) et conclusions, où cette dualité est clairement supposée.

35. Cf. Discours cit., supra, note 22 plus particulièrement parties 3 et 4 . 


\section{LES RÈGLES DU CODE ET LES DIFFÉRENTS STANDARDS DE BONNE ADMINISTRATION}

Ces différents niveaux de bonne administration sont représentés dans le contenu du code. Premièrement, celui-ci opère une codification des principes généraux de droit administratif européen (légalité, non-discrimination, proportionnalité, absence d'abus de pouvoir, respect des attentes légitimes et transparence) ${ }^{36}$. Deuxièmement, il rappelle les droits et obligations procédurales et substantielles qui découlent de règles expresses du droit communautaire. Parmi ceux-ci, on retrouve des droits fondamentaux contenus dans la Charte (protection des données personnelles, le droit de réclamation auprès du Médiateur européen) ${ }^{37}$. On retrouve également des droits énumérés à l'article 41 (le droit de voir ses affaires traitées impartialement, équitablement et dans un délai raisonnable; le droit d'être entendu et de faire des déclarations; l'obligation de motiver les décisions; les droits linguistiques) ${ }^{38}$. Certains, enfin, correspondent grosso modo à des règles primaires du droit de l'Union européenne, même s'ils sont rédigés avec plus de précision dans le code pour mettre l'accent sur la protection procédurale (notification des décisions) ${ }^{39}$. Un troisième niveau englobe des règles de pratique administrative inspirées par l'idée de la prestation d'un bon service au public. En principe, celles-ci ne constituent pas des droits ou règles invocables devant un juge (l'obligation d'informer le public sur le traitement des affaires, d'agir avec courtoisie, d'accuser réception d'une lettre ou d'une plainte et de fournir des informations sur le fonctionnaire et le service en charge de la question, de transférer le dossier au service compétent, d'indiquer les voies d'appel possibles, ainsi que des règles sur la façon de gérer des demandes et d'organiser des archives) ${ }^{40}$.

Les codes des institutions et organes européens qui ont suivi l'adoption du code contiennent également des principes juridiques, des droits subjectifs, des règles extrajuridiques, mais ils sont souvent rédigés en des termes différents et sont fréquemment moins complets que celui proposé par le Médiateur ${ }^{41}$.

36. Articles 4 à 7 et 10 (2). Les dispositions sur la transparence renvoient pour l'essentiel au règlement sur l'accès aux documents (article 23) et à la publicité du code lui-même (article 25).

37. Articles 8 et 43 de la Charte.

38. On peut estimer que les articles 8 (impartialité et indépendance), 9 (objectivité) et 11 (équité) renvoient aux différentes dimensions du traitement juste et équitable; l'article 17 est en lien avec le droit d'avoir ses affaires traitées dans un délai raisonnable; les autres droits et obligations étant contenues respectivement dans les articles 16,18 et 13 .

39. Article 20 (1) du code et article 254 (3) CE (voir l'article 297 (2), troisième paragraphe du Traité sur le fonctionnement de l'Union européenne).

40. Respectivement articles 10 (3), 12, 14, 15, 19, 22 et 24.

41. Il y a en fait des différences importantes entre le code proposé par le Médiateur et ceux adoptés respectivement par le Parlement européen, la Commission et le Secrétaire général du Conseil. Voir Guide sur les obligations des fonctionnaires et agents du Parlement européen (code de bonne conduite) (JOCE C 97/1, 5 avril 2000); annexe à la décision de la Commission du 17 octobre 2000 modifiant son règlement intérieur (JOCE L 267/63, 20 octobre 2000) ; décision du Secrétaire général du Conseil/Haut représentant pour la politique étrangère et de sécurité commune du 25 juin 2001 établissant un code de bonne conduite administrative pour le secrétariat général du Conseil de l'Union européenne et de son personnel dans leurs relations professionnelles avec le public, Partie III (JO C 189/1, 5.7.2001). Notons que certaines agences ont pris pour modèle le code de la Commission plutôt que celui proposé par le Médiateur (par exemple, décision $\mathrm{n}^{\circ}$ ADM-00-37 du Président de l'Office [de l'harmonisation dans le marché intérieur] du 9 juillet 2001 sur l'adoption d'un code de bonne conduite, disponible à http://oami.europa.eu/fr/office/aspects/decisions/adm00-37.htm). 


\section{Principes généraux, règles procédurales et substantielles}

Il convient de souligner deux aspects en lien avec les deux premiers niveaux mentionnés. Tout d'abord, l'inclusion dans le code de principes généraux du droit communautaire et de règles procédurales et substantielles qui avaient déjà été mentionnées à d'autres endroits répond à un objectif spécifique. Elle renforce l'idée défendue par le Médiateur dès l'origine selon laquelle l'évaluation des cas de mauvaise administration - le terme qui delimite le mandat du Médiateur en vertu de l'article 195 Traité CE (228 TFUE) - entraîne forcément une évaluation de la légalité des actions des institutions ${ }^{42}$. Notons qu'au moment où le Médiateur commença, de sa propre initiative, une enquête sur l'existence et la disponibilité de codes de bonne conduite administrative des institutions et organes européens (novembre 1998) et plus tard lorsqu'il proposa le code sous la forme d'un projet de recommandation (juillet 1999), cette approche du concept de mauvaise administration avait été contestée par la Commission dans deux procédures de plainte examinées par le Médiateur ${ }^{43}$. Rappeler que les standards de conduite administrative incluaient le respect des règles et procédures de nature juridique n'était pas seulement cohérent avec le concept de mauvaise administration tel qu'il était conçu par le Médiateur, mais venait également renforcer cette position, du fait de la vocation du code à influencer les codes de bonne conduite adoptés par les institutions et les organes de l'Union européenne. Et c'est ce qui s'est produit, même si tous ces principes et règles ne sont pas nécessairement reproduits dans tous les codes.

Deuxièmement, même si presque toutes les règles de la première et de la seconde catégorie correspondent à des règles et principes européens déjà contenus ailleurs - plus particulièrement dans la jurisprudence des juridictions européennes - les dispositions du code ne sont pas toujours une simple reformulation. Tout d'abord, la plupart des règles sont rédigées comme des obligations incombant aux fonctionnaires plutôt qu'aux institutions dont ils relèvent ${ }^{44}$. Cette distinction n'a pas en soi de conséquences juridiques directes, mais reste intéressante. Bien sûr, cela ne veut pas dire que les institutions elles-mêmes ne sont pas liées par les obligations contenues dans le code : ce serait non seulement illogique, mais également contraire à la prescription de l'article premier de celui-ci ${ }^{45}$. Cela ne peut pas non plus avoir pour conséquence, comme on pourrait le penser, que le code transpose par la sorte ces règles à l'activité interne de l'administration européenne, régulant ainsi les

42. Voir $R A M E, 1995$, pp. 8-9 ; RA ME, 1997, pp. 21-27. Le Médiateur y propose la définition suivante de la mauvaise administration : «Il y a mauvaise administration lorsqu'un organisme public n'agit pas en conformité avec une règle ou un principe ayant pour lui force obligatoire ». Par ailleurs, il précise que si le rôle du Médiateur n'est pas limité à l'évaluation de la légalité d'actes administratifs, il inclut néanmoins cet aspect («Lorsque le Médiateur européen examine la conformité de l'action d'une institution ou d'un organe communautaire avec les règles et les principes qui s'imposent à cette institution ou à cet organe, il lui incombe tout d'abord - et c'est d'ailleurs sa tâche essentielle - de vérifier si le droit a été respecté », RA ME, 1997 , pp. 25-26). Il en découle que la définition ci-dessus inclut des règles et principes de nature juridique, sans être limitées à ceux-ci.

43. Décision sur la plainte 449/96/20.02.96/HKC/PD contre la Commission européenne (RA ME, 1998, pp. 46-48) et décision sur la plainte Q5/98/IJH - OI/3/99/IJH, contre la Commission européenne (RA ME, 1999, pp. 19-21).

44. Le terme «fonctionnaire » inclut les fonctionnaires et autres agents soumis au statut et au régime applicable aux autres agents, ainsi qu'aux autres personnes travaillant pour les Communautés européennes (article 2 (1), (2) et (4) (b) du code). En conformité avec la définition du code (article 2 (4) (a)), le terme d' « institution » recouvre à la fois les institutions et les organes.

45. «Dans leurs relations avec le public, les institutions et leurs fonctionnaires respectent les principes énoncés dans le présent code ». La référence expresse aux institutions dans cet article résulte d'un amendement proposé par le Parlement européen au projet du Médiateur (Voir Résolution A5-0245/2001, cit., note 12). 
rapports entre les fonctionnaires et les institutions ${ }^{46}$. Cela doit plutôt être considéré comme favorisant un sentiment d'appropriation des décisions rendues par chaque fonctionnaire et des actions entreprises dans leur rapport avec le public, qui seront reflétées dans les décisions et actions des institutions auxquelles ils appartiennent. En même temps, il faut lire cela en lien avec l'article 3(1) du code, selon lequel les principes qui y sont définis s'appliquent à toutes les relations entre les institutions et le public. En ce sens, la focalisation sur les fonctionnaires comme sujets de la plupart des règles du code illustre le fait que les standards de bonne pratique administrative définis dans le code ne s'appliquent pas seulement aux procédures qui conduisent à l'adoption par les institutions d'actes formels, mais « de façon générale à l'activité des administrations », comme par exemple à la diffusion d'information concernant les activités de l'institution ${ }^{47}$.

De plus, certaines des dispositions du code établissent des règles qui prolongent le contenu de garanties juridiques déjà reconnues aux personnes dans leurs relations avec l'administration européenne. C'est notamment le cas pour le droit d'être entendu. Tels qu'ils sont formulés dans le code, les droits de la défense - et donc aussi le droit d'être entendu - sont reconnus lorsque «les droits ou intérêts des citoyens sont en jeu » alors que dans la jurisprudence le droit d'être entendu est reconnu dans toute procédure susceptible d'aboutir à un acte faisant grief à une personne déterminée, en règle générale, à la personne à l'encontre de laquelle la procédure est ouverte ${ }^{48}$. Par ailleurs, ce droit doit être garanti pour «tout membre du public» dans les cas où une décision affecte ses «droits ou intérêts » ${ }^{49}$. De surcroît, selon le code, les droits de la défense doivent être garantis «à chaque étape de la procédure », ce qui implique que la personne puisse suivre de façon quasi-continue le développement du processus décisionnel. Cette solution contredit certaines arrêts se prononçant dans le cadre de procédures complexes dans lesquels les tribunaux européens avaient admis que, même si une étape de la procédure est développée devant la Commission, celle-ci n'a pas forcément le devoir d'assurer devant elle-même le droit d'être entendu ${ }^{50}$. Enfin, les limites de la règle peuvent être interprétées différemment, selon que l'on considère que les « décisions » renvoient soit à des décisions individuelles, soit à des actes adoptés sous l'une des formes prévues à l'article 249 du Traité CE (288 TFUE) ou, de façon plus imprécise, à tout acte pouvant avoir des effets négatifs, en

46. Article 3 (2) du code.

47. Voir Draft recommendation to the European Anti-Fraud Office (OLAF) in plainte 1840/2002/GG, 18 juin 2003, pt 1.5. Cette plainte est résumée dans le $R A M E, 2003$, pp. 183-185.

48. Article 16 (1) du code. Soulignant également la formulation plus large de la Charte, Ka\&nacute ;ska, op. cit., p. 318. Cette disposition renvoie aux droits de la défense, mais au vu du titre de l'article, il est vraisemblable que l'utilisation de ce terme puisse être comprise comme une synecdoque. V. parmi d'autres arrêts, CJCE, 29 juin 1994, Fiskano c. Commission aff. C-135/92, Rec. p. I-2885, pt 39 et plus récemment, TPI, 11 juillet 2007, Alrosa Company Ltd c. Commission, aff. T-170/06, Rec. p. II-2601, pt 191. On peut trouver l'origine d'une formulation plus large du droit d'être en entendu dans l'arrêt Transocean, où il est reconnu aux « destinataires de décisions des autorités publiques qui affectent de manière sensible leurs intérêts » (CJCE, 23 octobre 1974, Transocean Marine Paint Association c. Commission, aff. 17/74, Rec. p. 1063, pt 15, nos italiques). Cette formulation a cependant eu moins d'influence dans la jurisprudence, comme le montre la formulation de l'article 41(2) al 1 de la Charte.

49. Article 16 (2) du code.

50. Article 16 (1) du code. Voir TPI, 9 novembre 1995, France-aviation c. Commission, aff. T-346/94, Rec. p. II-2841, pt 36 ; TPI, 18 juillet 2007, Ente per le Ville vesuviane c. Commission, aff. T-189/02, Rec. p. II-89, pts 91, 93-100 (dans cette affaire qui concernait l'usage de fonds structurels, le Tribunal n'a pas considéré la question de savoir si la personne aurait dû être entendue directement par la Commission et non pas seulement pas l'État membre concerné). 
conformité avec la disposition générale du code selon laquelle ses dispositions s'appliquent à toutes les relations entre les institutions et le public ${ }^{51}$.

L'obligation de motiver a également un champ d'application différent dans les règles existantes et dans les dispositions du code. Les dispositions du code limitent l'obligation aux décisions des institutions qui pourraient avoir un effet négatif sur les droits et les intérêts de personnes privées mais, en individualisant seulement cet aspect de l'obligation, il l'étend également. En effet, selon le code, si un raisonnement détaillé n'est pas possible en raison du trop grand nombre de personnes concernées par des décisions similaires, un citoyen qui en fait expressément la demande aura tout de même droit à une réponse motivée individuelle ${ }^{52}$. À notre connaissance, les juridictions communautaires n'ont pas étendu à ce point le rôle protecteur de l'obligation de motivation ${ }^{53}$. Lu comme tel, la disposition du code ne doit pas être perçue comme restreignant la règle contenue dans l'article $253 \mathrm{du}$ Traité CE (voir aussi l'article 296 du TFUE), mais plutôt comme prolongeant l'un de ses aspects, le même qui a aussi été individualisé à l'article 41(2) al. c) de la Charte ${ }^{54}$.

Le contenu différent de certaines des règles du code par rapport au droit existant pourrait être une indication importante pour que les plaignants éventuels décident quelle voie de contrôle suivre, en fonction des circonstances de leur affaire et des compensations possibles : action judiciaire ou plainte au Médiateur. Un champ plus large ou une fonction protectrice plus forte des règles du code pourraient indiquer aux plaignants que la perception du Médiateur sur l'application de certaines garanties procédurales serait plus favorable à leurs intérêts que la position des tribunaux européens.

Au contraire, dans la mesure où le champ d'application des dispositions du code est le même que celui des principes et des règles précédemment établies dans des sources juridiquement contraignantes, il est peu probable que leur inclusion dans le code n'ait d'autre valeur ajoutée juridique que celle d'éclaircir quels principes peuvent être associés au principe de bonne administration.

\section{Règles de comportement éthique et bon service administratif}

Comme indiqué précédemment, la bonne administration comprend aussi des règles de comportement éthique dans l'exercice de la fonction publique ainsi que des obligations ayant trait au bon fonctionnement du service administratif, le respect de celles-ci émanant

51. Ce doute ne se justifie pas pour l'article 41(2), où la référence aux «mesures individuelles » à l'alinéa a) indique que le sens du mot « décision » dans l'alinéa c) devrait probablement avoir le même sens. Sur l'ambiguïté du terme de «décision » en droit communautaire, voir Von Bogdandy (Armin) et al,. «Legal instruments in European Union Law and their reform: a systematic approach and an empirical analysis », Yearbook of European Law, Vol. 23, 2004, pp. 91-136, pp. 101-106.

52. Article 18 (1) et (3) du code.

53. En principe, l'institution ou l'organe prenant la décision n'a pas à prendre en considération tous les éléments factuels ou juridiques qui ont été soulevés par une personne intéressée pendant la procédure administrative (TPI, 11 décembre 1996, Van Megen Sports Group BV c. Commission, aff. T-49/95, Rec. p. II-1799, pt 55 ; TPI, 21 mars 2002, Colin Joynson c. Commission, aff. T-231/99, Rec. p. II-2085, pt 166 ; CJCE, 30 septembre 2003, Germany c. Commission, aff. C-301/96, Rec. p. I-9919, pt 140) et les raisons avancées ont seulement besoin de «fournir à l'intéressé une indication suffisante pour savoir si la décision est bien fondée ou si elle est éventuellement entachée d'un vice permettant d'en contester la validité » (Van Megen, cit., pt 51). Ceci a pour but d'éviter ou de limiter l'annulation de saines décisions pour des questions de formalités : voir Lenaerts (Koen) et Vanhamme (Jan), « Procedural rights of private parties in the Community administrative process », Common Market Law Review, Vol. 34, 1997, pp. 531-569, pp. 563-564.

54. Clairement, l'usage abusif de cette règle doit être évité par analogie avec ce qui est prévu à l'article 14(3) du code : «L'envoi d'un accusé de réception et d'une réponse n'est pas nécessaire lorsque les lettres ou les requêtes sont en nombre excessif ou lorsqu'elles revêtent un caractère répétitif ou inapproprié. » 
davantage d'un certain sens de la «culture du service public » que d'une obligation légale ${ }^{55}$. Tel est le cas pour les obligations des fonctionnaires d'être consciencieux, disponible dans leurs relations avec le public ou de s'excuser en cas d'erreur portant préjudice aux droits ou aux intérêts d'une personne ${ }^{56}$.

Certes, la frontière n'est pas toujours facile à tracer entre des obligations légales et des obligations éthiques extra-juridiques qui tiennent plus de la conscience professionnelle. Comme nous avons pu le souligner auparavant, les règles de bonne administration peuvent recouvrir des aspects à la fois juridiques et extra-juridiques. Dans tous les cas, en droit européen, les règles du code qui ne correspondent pas à des principes juridiques ou à des droits procéduraux et substantiels reconnus ne sont pas, en principe, des règles contraignantes de bonne pratique administrative. Elles sont seulement consacrées dans des codes de bonne conduite - celui proposé par le Médiateur et ceux adoptés par les institutions et organes européens - qui sont publiés dans la série C du Journal officiel ou seulement sur le site internet des agences.

En règle générale, ce sont des mesures internes qui peuvent être considérées comme contraignantes pour leurs auteurs sur la base du principe patere legem quam ipse fecisti. Ce constat vaut pour le code applicable aux fonctionnaires du Conseil, ainsi que pour le code de conduite du Parlement européen. Le premier, tout en indiquant que les fonctionnaires du Conseil doivent respecter les dispositions du code, précise que ce respect doit être assuré en interne ${ }^{57}$. De plus, la décision adoptée par le Secrétaire général du Conseil exclut explicitement l'idée que les règles du code puissent être interprétées comme créant des droits additionnels : leur objectif est simplement de faciliter la mise en œuvre de droits et obligations issues du Traité et du droit dérivé ${ }^{58}$. Le second, qui comprend les règles sur les obligations générales et de service des fonctionnaires et autres agents, exclut explicitement tout effet contraignant : il constitue un «mode d'emploi » et fournit une « référence éthique aux fonctionnaires et autres détenteurs d'un mandat public » ${ }^{59}$.

Le code de la Commission est le seul à être publié dans la série L du Journal officiel des Communautés européennes. Il est rédigé en des termes qui affirment sa nature contraignante 60 et il est incorporé comme annexe aux règles de procédure de la Commission, partageant ainsi ses effets obligatoires. En conséquence, ces règles peuvent être invoquées par les personnes physiques et morales dans la mesure où elles visent à protéger les individus et pas seulement à régir l'organisation du fonctionnement interne du service dans l'intérêt d'une bonne administration ${ }^{61}$. Alors que le code proposé par le Médiateur contient des règles des deux natures, le code de la Commission tend à limiter le

55. L'expression «culture du service public » est utilisée dans les $R A M E$ et dans le discours cit., note 26 .

56. Article 12 (1) et (3) du code.

57. Voir article 3 de la décision et article premier du code (annexé à la décision). Pour les références complètes, voir note 42. Cela s'applique évidemment au code dans son ensemble - qui est considérablement plus court que celui proposé par le Médiateur - mais qui est particulièrement pertinent en ce qui concerne les règles de cette catégorie.

58. Article 3 de la décision, cit., note 41.

59. code, cit., note 41, point 4, p. 3, citant le second rapport du comité d'experts indépendants en soutien de cette approche.

60. Voir les dispositions sur le champ d'application du code (références note 42). Voir les observations sur une ancienne version de ce code du Médiateur dans son Rapport Spécial cit, note 9, Partie C.

61. CJCE, 7 mai 1991, Nakajima All Precision Co. Ltd c. Council, aff. C-69/89, Rec. p. I-2069, pt 49 et 50 ; TPI, 27 février 1992, BASF AG and others $c$. Commission, aff. jointes T-79/89, T-84/89, T-85/89, T-86/89, T-89/89, T-91/89, T-92/89, T-94/89, T-96/89, T-98/89, T-102/89 et T-104/89, Rec. p. II-315, pt 78. 
champ des règles qui pourraient être considérées comme ayant l'intention de protéger les individus ${ }^{62}$.

\section{LA FONCTION ET LES PERSPECTIVES DU CODE}

Le code a atteint partialement son objectif immédiat, dans la mesure où la Commission, le Conseil et le Parlement ont adopté des codes de bonne conduite administrative (bien qu'avec des contenus différents) et de nombreuses agences européennes se sont servies soit du code du Médiateur soit du code de la Commission comme modèle pour leur propre code. Son objectif ultime, c'est-à-dire l'adoption d'une loi établissant des règles communes de conduite pour les institutions et organes communautaires, n'a cependant pas été atteint et il ne semble pas qu'il le sera, en tout cas dans un futur proche. Dans ce contexte, quel est la pertinence actuelle du code, au-delà de sa contribution à clarifier le concept de bonne administration, comme nous l'avons évoqué plus haut ?

Tout d'abord, le code annonce la contribution du Médiateur au respect de règles et de principes précédemment établis ainsi qu'à l'établissement de «nouveaux » standards de bonne conduite administrative visant à la promotion de la conscience de service. En ce sens, il remplit certains des objectifs qui avaient justifié son adoption ${ }^{63}$. En effet, il est un indicateur utile pour les institutions et organes européens ainsi que pour le public sur les actes susceptibles d'être sanctionnés par le Médiateur. Les plaignants se fondent effectivement sur le code dans les affaires qu'ils portent devant le Médiateur et les institutions et organes convoqués par lui. Cependant, un aperçu de l' « usage quantitatif » du code dans les décisions et projets de recommandations du Médiateur semble indiquer que le recours au code n'est pas nécessairement la norme. Entre janvier 2001, l'année où le Médiateur a transmis son rapport spécial au Parlement sur l'adoption de codes de bonne conduite administrative, et juin 2009, seulement 320 décisions (sur 1319) et 23 projets de recommandations (sur 99) émis dans des affaires de mauvaise administration invoquent le code ${ }^{64}$. En outre, certains éléments portent à croire que les institutions n'accepteraient que les règles contenues dans leur propre code. Par exemple, la Commission a précisé qu'à son avis seul son propre code est contraignant à son égard alors qu'il s'agissait d'une affaire dans laquelle cela n'aurait rien changé au résultat, les règles en question étant présentes dans les deux codes ${ }^{65}$. De plus, notons que le code n'est pas toujours entièrement partagé par les institutions et organes. Les codes de conduite propres à ces derniers oscillent entre, d'une part, la reproduction du code rédigé par le Médiateur et la reconnaissance de celui-ci comme leur source principale - tel est le cas pour le code de quelques agences, même si la plupart ont préféré reproduire le code de la Commission - et, d'autre part, l'omission de

62. Voir l'article 22 du code et article 4 du code de la Commission, ainsi que les articles 16,18 et $19 \mathrm{du}$ code et l'article 3 du code de la Commission.

63. Voir code, Avant-propos du Médiateur européen, version en ligne (note 5), p. 4.

64. Cette statistique est le résultat d'une recherche, en anglais, dans la base de données du site du Médiateur, utilisant comme filtres les termes de «maladministration » et «code of good administrative behaviour ». Les résultats pour «maladministration» peuvent être un indicateur du nombre total de décisions et de projets de recommandations. Les résultats pour « code of good administrative behaviour » ne distinguent pas entre les renvois au code ou à d'autres codes adoptés par les institutions et organes, ni s'ils sont invoqués par les plaignants ou le Médiateur. Ils donnent cependant une indication générale sur l'utilisation du code.

65. Décision sur la plainte 3398/2005/ELB contre la Commission européenne, 29 décembre 2006, voir la plainte et les observations de la Commissions sur les observations du plaignant (Partie 2). 
nombreuses règles du code et de toute référence à celui-ci ${ }^{66}$. Le fait qu'en décembre 2008 seulement deux institutions avaient déclaré, en invoquant l'article 27, avoir mis en œuvre le code pourrait être un indice de la faible priorité accordée au code par les institutions européennes ${ }^{67}$.

Ensuite, le code indique des règles de bonne pratique administrative qui, bien que n'ayant pas un contenu juridique direct, peuvent devenir juridiquement pertinentes dans la mesure où leur violation peut affecter des droits procéduraux reconnus en droit européen 68 .

Par ailleurs, d'un point de vue normatif, le code pourrait esquisser de possibles développements du droit administratif européen, en particulier en ce qui concerne les règles dont la seule source dans les textes européens est dans le code et les décisions du Médiateur. De fait, le code trouve son inspiration dans le droit administratif des États membres - à la fois codes et lois de procédure administrative nationaux, et guides nationaux de pratique administrative - et dans des textes juridiques internationaux ${ }^{69}$. On peut soutenir que cette caractéristique en fait une source privilégiée pour des développements normatifs futurs. En particulier, sur la base du principe de bonne administration, les tribunaux européens peuvent considérer que certaines de ces règles deviennent des normes juridiques, dans la mesure où elles sont partagées par des États membres et dans la mesure où, dans des contextes politico-juridiques nouveaux, elles peuvent être juridiquement pertinentes pour résoudre des problèmes spécifiques en droit communautaire ${ }^{70}$.

En l'occurrence, des règles du troisième niveau telles qu'évoquées plus haut sont des règles juridiques contraignantes dans certains systèmes nationaux. Certaines sont incluses dans les obligations d'information et de conduite respectueuse auxquelles sont tenus les fonctionnaires dans l'exercice de leurs fonctions ${ }^{71}$. Ces obligations, que la loi estime comme inhérentes à l'exercice de la fonction publique, font partie du statut disciplinaire spécifique applicable aux employés publics et leur respect est assuré par des actions

66. Voir le code de l'Autorité européenne de sécurité des aliments (http://www.efsa.europa.eu/EFSA/ efsa_locale-1178620753812_1178620791688.htm) pour la première catégorie. Les codes évoqués dans la note 41 sont des exemples de la seconde catégorie.

67. Rapport présenté par Marta Hirsch-Ziembi\&nacute ;ska, avocat au bureau du Médiateur européen sur «The application of the European code of Good Administrative Behaviour by the European institutions » à la conférence In pursuit of good administration, Conseil de l'Europe en coopération avec la faculté de droit et d'administration de l'université de Varsovie, 29-30 novembre 2007 (http://www.coe.int/t/e/legal_affairs/ legal_co-operation/administrative_law_and_justice/conferences/DA-ba-Conf \%20_2007_\%209\%20e \%20-

$\% 20$ M. \%20Hirsch-Ziembinska.pdf). Cependant, ce rapport donne une interprétation différente de ce fait: selon l'auteur, le petit nombre de commentaires reçus indiquerait plutôt que « la mise en œuvre des principes du code n'avait pas donné lieu à des difficultés » (p. 9).

68. Voir TPI, 11 mars 1999, Ensidesa c. Commission, aff. T-157/94, Rec. p. II-707, pts 24 et 25, et Dynamiki, cit. (note 29) pt 159, a contrario sensu.

69. Rapport spécial, cit. (note 8), Conclusion et recommandation $n^{\circ} 2$, note 9.

70. Sur le processus de réception de règles et de principes juridiques en droit communautaire, voir, parmi d'autres, Pescatore (Pierre), «Le recours, dans la jurisprudence de la Cour de justice des Communautés européennes, à des normes déduites de la comparaison des droits des États membres », Revue internationale de droit comparé, Vol. 32(2), 1980, pp. 337-359.

71. Au Portugal, voir l'article 3 (6) et (10) du Statut disciplinaire des travailleurs exerçant une fonction publique (Loi n ${ }^{\circ} 58 / 2008,9$ septembre, DR [Diário da República] I, n 174). Dans le système espagnol, le manque de considération ou le comportement inadéquat à l'encontre des personnes ayant affaire à l'administration peut constituer une violation des obligations des employés publics (articles 7, o) et 8, c) du «Régime disciplinaire des fonctionnaires »-décret royal n 33/1986, 10 janvier, BOE [Boletín Oficial] n ${ }^{\circ} 15$, toujours en vigueur après l'adoption de la loi $\mathrm{n}^{\circ} 7 / 2007,12$ avril, BOE $\mathrm{n}^{\circ} 89$; voir aussi les principes de conduite consacrés à l'article 54 (1) et (4) de cette loi). Curieusement, le fait d'être traité « avec respect et déférence par les autorités et les officiels », ce qui pourrait être considéré comme équivalant au fait d'être traité avec courtoisie, est aussi un droit du citoyen (article 35 (i) de la loi n ${ }^{\circ}$ 30/1992, 26 novembre, BOE $n^{\circ} 23$ ). 
disciplinaires ${ }^{72}$. Au Royaume-Uni, certaines de ces règles proviennent du code du service public, comme l'obligation de rectifier promptement des erreurs, qui doivent être incorporées dans les conditions de services par les services administratifs et agences ${ }^{73}$. D'autres obligations administratives, comme celle d'archiver les courriers et documents, ou les règles sur le transfert d'un courrier ou d'une plainte au service compétent, sont des règles générales de la procédure administrative ${ }^{74}$. Dans certains systèmes, d'autres règles de ce troisième niveau sont rédigées sous la forme de droits ou de facultés pour les citoyens qui sont légalement protégés par des actes ayant force obligatoire. Tel est le cas pour le droit de recevoir ou d'obtenir un accusé de réception ${ }^{75}$, le droit de savoir quel service est en charge de l'affaire le ou la concernant ${ }^{76}$, le droit d'être informé, lorsque c'est nécessaire, sur la procédure à suivre dans une situation particulière ${ }^{77}$.

Enfin, le code peut indiquer des voies possibles au développement de certains des droits procéduraux contenus dans l'article 41 de la Charte, particulièrement si celle-ci acquiert une force obligatoire par l'entrée en vigueur du traité de Lisbonne et si, en conséquence, les juridictions européennes deviennent plus enclines à recourir à cette disposition et à promouvoir de nouvelles évolutions. Comme précisé précédemment, les dispositions du code sur le droit d'être entendu et l'obligation de motivation étendent le contenu de ces garanties au-delà de ce qui est légalement envisagé. Pour le moment, les dispositions du code pour lesquelles le rôle protecteur de ces droits et obligations est renforcé, ne peuvent être prises en compte qu'en tant que dimensions extra-juridiques de ces garanties procédurales telles qu'elles sont suggérées par le Médiateur et pas nécessairement partagées par les institutions ${ }^{78}$. Le choix de décider si ces règles peuvent, ou doivent, passer le «filtre juridique » appartient en définitive aux acteurs juridiques qui décideront si certains intérêts devraient être juridiquement protégés et si certains comportements sont susceptibles de les affecter de manière socialement pertinente. Ce choix est fait à la lumière des caractéristiques et des besoins du système politico-juridique. La nature

72. Les statuts particuliers d'employés publics ont survécu aux réformes qui ont affecté le service public dans de nombreux pays européens au cours des deux dernières décennies et qui visaient, notamment, à l'harmonisation entre le statut des fonctionnaires et les contrats de travail des salariés du secteur privé : voir Demmke (Christoph), European civil services between tradition and reform, EIPA, Maastricht, 2004, p. 95.

73. Règle 4.1.5 du code de Gestion du Service Public (http://www.civilservice.gov.uk/iam/codes/csmc/ index.asp) et règle $7 \mathrm{du}$ code du Service Public (http://www.civilservice.gov.uk/iam/codes/cscode/index.asp). Soulignons que ce code vise à assurer l'efficacité et le bon comportement des services et ne se concentre donc pas sur l'amélioration des relations avec le public.

74. Sur la première règle, voir article 80 , décret-loi $\mathrm{n}^{\circ} 442 / 91,15$ novembre, $D R \mathrm{n}^{\circ} 263$, I-A, amendé (Portugal) ; article 38, loi $\mathrm{n}^{\circ} 30 / 1992$, cit. (Espagne). Sur la seconde règle voir, article 34, décret-loi $\mathrm{n}^{\circ} 442 / 91$ (Portugal) ; article 20, loi $\mathrm{n}^{\circ}$ 2000-321, du 12 avril 2000 relative aux droits des citoyens dans leurs relations avec l'administration, Journal Officiel (France) ; article 20, loi n $30 / 1992$, cit. (Espagne) ; article 6 (1) (e), loi $\mathrm{n}^{\circ}$ 241, 7 août 1990, Gazzetta Ufficiale n 192, amendée (Italie; quoique se référant à l'adoption d'actes formels).

75. Article 19, loi n 2000-321, cit. (France); article 70 (3), loi 30/1992, cit. ; article 81, Décret-loi $\mathrm{n}^{\circ} 442 / 91$, cit. (Portugal). Cf. article 14 (1) du code.

76. Article 61 (2), décret-loi no 442/91 (Portugal); article 35 (b), loi no 30/1992, (Espagne); article 4, loi $\mathrm{n}^{\mathrm{o}} 2000-321$ (France) ; article 5 (3), loi no 241/1990 (Italie).

77. Articles 7 (1) (a) et 61 (2), décret-loi no 442/91 (Portugal); article 35 (g), loi 30/1992 (Espagne); article 10-bis, loi n 241/1990 (Italie; limitée à certains types de procédures).

78. Par exemple, le code de la Commission s'écarte de l'approche plus «proactive » du code en matière du droit d'être entendu : ses fonctionnaires doivent respecter ce droit, « lorsque le droit communautaire prévoit que les parties intéressées doivent être entendues » (point 3 du code de la Commission, cit., note 42, sous le titre « écouter toutes les parties ayant un intérêt direct »). On retrouve cette formulation à l'article 16 du code adopté par l'Agence européenne des produits chimiques (http://echa.europa.eu/doc/FINAL_MB_11_2008_ code_of_Good_Conduct.pdf). 
composite du concept de bonne administration et ses objectifs programmatiques indiquent les limites d'un éventuel processus de «juridicisation».

$$
\text { * * }
$$

L'aperçu des vicissitudes du code et de la genèse du droit à une bonne administration consacré dans la Charte montre que l'existence de l'article 41 est étroitement liée à l'adoption du code. Cependant, l'analyse du concept de bonne administration et du contenu du code a démontré que ce dernier ne peut être interprété comme explicitant le contenu du droit à une bonne administration. Quelle que soit l'étendue de son champ d'application concret à l'avenir, l'article 41 pose la bonne administration comme un droit public subjectif, alors qu'une partie des règles du code peuvent difficilement être considérées comme visant principalement la protection des individus dans leurs relations avec l'administration européenne. En outre, le code précise le contenu de la bonne administration dans la mesure où il souligne les ramifications juridiques et extra-juridiques du concept, mettant ainsi l'accent sur sa particularité : la combinaison et les continuités entre ses dimensions juridiques et extra-juridiques. En particulier, il rappelle que l'administration devrait s'efforcer de promouvoir certains aspects de la bonne pratique administrative qui vont au-delà de la sphère juridique au sens strict. Sont ainsi concernées les dimensions extra-juridiques des garanties procédurales juridiquement protégées, tel que le droit d'être entendu et l'obligation de motivation. En ce sens, séparer le code de l'article 41 - et donc ignorer leur enchevêtrement initial - est une étape importante pour la compréhension de l'apport du code au développement du droit administratif européen. En effet, indépendamment de la réalisation des objectifs annoncés du code - son adoption par les institutions européennes et son adoption sous la forme d'un acte ayant juridiquement force obligatoire - il permet de montrer des voies possibles de développement juridique de la bonne administration. 